\title{
A periodic boundary value problem for nonlinear singular differential systems with 'maxima'
}

\author{
Peiguang Wang ${ }^{1 *}$ (D) and Xiang Liu²
}

\section{"Correspondence:}

pgwang@hbu.edu.cn

${ }^{1}$ College of Electronic and

Information Engineering, Hebei

University, Baoding, 071002, China

Full list of author information is

available at the end of the article

\begin{abstract}
In this paper, by using the method of quasilinearization to discuss the periodic boundary value problem for a nonlinear singular differential system with 'maxima', we obtain monotone iterative sequences of approximate solutions which converge uniformly and quadratically to the solution of the nonlinear singular differential system with 'maxima'.
\end{abstract}

MSC: 34D20; 34A37

Keywords: singular system; maxima; quasilinearization

\section{Introduction}

Convergence has an important role to play in the development of qualitative theory of various nonlinear system. The method of quasilinearization is a powerful technique for obtaining approximate solutions of nonlinear problems. A systematic development of the method to ordinary differential equations was provided by Bellman and Kalaba [1], Lakshmikantham and Vatsala [2]. Afterwards, some generalized results were achieved for various types of differential systems, referred to in the monographs $[3,4]$, for functional differential equations to $[5,6]$, for impulsive equations to $[7,8]$, for fractional differential equations to [9-11], and to the references cited therein.

Recently, singular differential systems introduced by Rosenbrock [12], are studied because they have many applications in practical fields, such as non-Newtonian fluid mechanics, optimal control problems, and electrical circuits and some population growth models. It is more complicated than the ordinary systems, and its qualitative analyses involve greater difficulty than those of the ordinary ones. We noticed that there were few applicable results of convergence to singular differential systems [13-16] or differential equations with 'maxima' [17, 18]. By using the method of quasilinearization, in [13], the authors investigated the uniform and quadratic convergence of the initial value problem for singular differential systems. Agarwal and Hristova [18] investigated the initial value problem for differential equations with 'maxima'. Singular differential systems with 'maxima' have not been studied yet.

In this paper, we apply the method to the study of the convergence of periodic boundary value problem (PBVP) of singular differential systems with 'maxima'. The arrangement of 
this paper is as follows. In Section 2, we prove some basic lemmas which are needed in succeeding sections. In Section 3, under suitable conditions, we prove quadratic convergence of monotone sequences to the solution of singular differential systems with 'maxima'. In Section 4, under less restrictive assumptions, we prove quadratic convergence by using the method of generalized quasilinearization.

\section{Some basic results}

Consider the periodic boundary value problem for the nonlinear singular differential system with 'maxima' (PBVP)

$$
\left\{\begin{array}{l}
A x^{\prime}(t)=f\left(t, x(t), \max _{s \in[t-h, t]} x(s)\right), \quad t \in[0, T] \\
x(0)=x(T), \quad x(t)=x(0), \quad t \in[-h, 0]
\end{array}\right.
$$

where $A$ is a singular $n \times n$ matrix, $x \in R^{n}, f \in C\left([0, T] \times R^{n} \times R^{n}, R^{n}\right), h$ and $T$ are positive constants.

Throughout this paper, the inequalities between vectors are understood component wise. Defining $|x|^{2}=\left(\left|x_{1}\right|^{2},\left|x_{2}\right|^{2}, \ldots,\left|x_{n}\right|^{2}\right)^{T}$ for $x \in R^{n},|A|=\left(\left|a_{i j}\right|\right)$ for $A \in R^{n \times n}$, and

$$
f_{x y} h k=\left(\sum_{i, j=1}^{n} \frac{\partial^{2} f_{1}}{\partial x_{i} \partial y_{j}} h_{i} k_{j}, \sum_{i, j=1}^{n} \frac{\partial^{2} f_{2}}{\partial x_{i} \partial y_{j}} h_{i} k_{j}, \ldots, \sum_{i, j=1}^{n} \frac{\partial^{2} f_{n}}{\partial x_{i} \partial y_{j}} h_{i} k_{j}\right)^{T}, \quad h, k \in R^{n}
$$

Let the functions $\alpha_{0}, \beta_{0} \in C\left([-h, T], R^{n}\right)$ be such that $\alpha_{0}(t) \leq \beta_{0}(t)$, we introduce two sets:

$$
\begin{aligned}
S\left(\alpha_{0}, \beta_{0}\right)= & \left\{u \in C\left([-h, T], R^{n}\right) \mid \alpha_{0}(t) \leq u(t) \leq \beta_{0}(t), t \in[-h, T]\right\}, \\
\Omega\left(\alpha_{0}, \beta_{0}\right)= & \left\{(t, x, y) \in[0, T] \times R^{n} \times R^{n} \mid \alpha_{0}(t) \leq x(t) \leq \beta_{0}(t),\right. \\
& \left.\max _{s \in[t-h, t]} \alpha_{0}(s) \leq y(t) \leq \max _{s \in[t-h, t]} \beta_{0}(s)\right\} .
\end{aligned}
$$

Definition 2.1 Let $\alpha_{0}, \beta_{0} \in C\left([-h, T], R^{n}\right) \cup C^{1}\left([0, T], R^{n}\right)$. The function $\alpha_{0}$ is called a lower solution of PBVP (2.1), if the following inequalities are satisfied:

$$
\left\{\begin{array}{l}
A \alpha_{0}^{\prime}(t) \leq f\left(t, \alpha_{0}(t), \max _{s \in[t-h, t]} \alpha_{0}(s)\right), \quad t \in[0, T] \\
\alpha_{0}(0) \leq \alpha_{0}(T), \quad \alpha_{0}(t)=\alpha_{0}(0), \quad t \in[-h, 0]
\end{array}\right.
$$

Analogously, the function $\beta_{0}$ is called an upper solution of PBVP (2.1), if the inequalities hold in an opposite direction.

Now, we will prove the following results which are needed for our further investigations. First, we consider the singular differential inequalities

$$
\left\{\begin{array}{l}
A x^{\prime}(t)+M(t) x(t) \leq 0, \quad t \in[0, T] \\
x(0) \leq x(T)
\end{array}\right.
$$

where $A, M(t)$ are $n \times n$ matrices, $A$ is singular and $M(t)$ is continuous on $[0, T]$. 
Lemma 2.1 Assume that the following conditions hold.

$\left(\mathrm{H}_{2.1}\right)$ There exists a constant $\lambda$ such that $L(t)=[\lambda A+M(t)]^{-1}$ exists, $\hat{A}=A L(t)$ is a constant matrix.

$\left(\mathrm{H}_{2.2}\right)$ There exists a nonsingular matrix $Q$ such that $Q^{-1}$ exists and $Q^{-1},(L Q) \geq 0$, satisfying

$$
Q^{-1} \hat{A} Q=\left(\begin{array}{ll}
C & 0 \\
0 & 0
\end{array}\right), \quad Q^{-1} \hat{M} Q=\left(\begin{array}{cc}
I_{1}-\lambda C & 0 \\
0 & I_{2}
\end{array}\right)
$$

where $C$ is a diagonal matrix with $C^{-1} \geq 0,\left(I_{1}-\lambda C\right) \leq 0, \hat{M}=M(t) L(t)=I-\lambda \hat{A}$.

$\left(\mathrm{H}_{2.3}\right)$ The matrix $D^{-1}=\left(I-e^{-\int_{0}^{T} C^{-1}\left(I_{1}-\lambda C\right) d s}\right)^{-1}$ exists and is positive.

Then $x(t) \leq 0$ on $[0, T]$.

Proof Using the transformation $x(t)=L(t) y(t)$, we get from (2.3)

$$
\hat{A} y^{\prime}+\hat{M} y \leq 0, \quad t \in[0, T] .
$$

Letting $y(t)=Q z(t)$ in (2.4) and multiplying (2.4) by $Q^{-1} \geq 0$, we have

$$
\left[\begin{array}{ll}
C & 0 \\
0 & 0
\end{array}\right]\left[\begin{array}{l}
z_{1}^{\prime} \\
z_{2}^{\prime}
\end{array}\right]+\left[\begin{array}{cc}
I_{1}-\lambda C & 0 \\
0 & I_{2}
\end{array}\right]\left[\begin{array}{l}
z_{1} \\
z_{2}
\end{array}\right] \leq 0
$$

which is equivalent to $C z_{1}^{\prime}+\left[I_{1}-\lambda C\right] z_{1} \leq 0$, and $z_{2} \leq 0$ on $t \in[0, T]$. We see from $C z_{1}^{\prime}+$ $\left[I_{1}-\lambda C\right] z_{1} \leq 0$ that

$$
z_{1}(t) \leq e^{-\int_{0}^{t} C^{-1}\left(I_{1}-\lambda C\right) d s} z_{1}(0) \leq e^{-\int_{0}^{t} C^{-1}\left(I_{1}-\lambda C\right) d s} z_{1}(T)
$$

Then

$$
\left(I-e^{-\int_{0}^{T} C^{-1}\left(I_{1}-\lambda C\right) d s}\right) z_{1}(T)=D z_{1}(T) \leq 0 .
$$

Since $D^{-1}$ is nonsingular and positive, we conclude that $z_{1}(T) \leq 0$. Thus, $z_{1}(t) \leq 0, t \in$ $[0, T]$. Due to the fact that $x=L Q z$ and $L Q \geq 0$, we have $x(t) \leq 0$ on $[0, T]$. The proof is complete.

For the boundary value problem

$$
A x^{\prime}+M(t) x=g(t), \quad E L^{-1}(0) x(0)-F L^{-1}(T) x(T)=\eta .
$$

We have the following well-known result.

Lemma 2.2 (See [14]) Assume that the condition $\left(\mathrm{H}_{2.1}\right)$ of Lemma 2.1 holds, index $(A)=1$, and

$\left(\mathrm{H}_{2.4}\right)$ the boundary condition satisfies the requirement that $J=E-F \exp \left\{-\hat{A}^{D} \hat{M} T\right\}$ is invertible. 
Then the unique solution of

$$
\hat{A} y^{\prime}+\hat{M} y=g(t), \quad E y(0)-F y(T)=\eta,
$$

is given by

$$
y(t)=e^{-\hat{A}^{D} \hat{M} t} \hat{A} \hat{A}^{D} J^{-1}\left(\eta-\xi_{1}\right)+\hat{A} \hat{A}^{D} \int_{0}^{T} G(t, \sigma) g(\sigma) d \sigma+\left(I-\hat{A} \hat{A}^{D}\right) \hat{M}^{D} g(t),
$$

where $\xi_{1}=E\left(I-\hat{A} \hat{A}^{D}\right) \hat{M}^{D} g(0)-F\left(I-\hat{A} \hat{A}^{D}\right) \hat{M}^{D} g(T)$,

$$
G(t, s)= \begin{cases}e^{-\hat{A}^{D} \hat{M} t} J^{-1} E e^{\hat{A}^{D} \hat{M} \sigma} \hat{A}^{D}, & t>\sigma, \\ e^{-\hat{A}^{D} \hat{M} t} J^{-1} F e^{-\hat{A}^{D} \hat{M}(T-\sigma)} \hat{A}^{D}, & t<\sigma,\end{cases}
$$

where $\hat{A}^{D}, \hat{M}^{D}$ mean the Drazin inverse of the matrices $\hat{A}, \hat{M}$. Note that once we have $y(t)$, we get $x(t)=L(t) y(t)$, where $x(t)$ is the solution of $(2.5)$.

Consider the singular differential inequalities

$$
\left\{\begin{array}{l}
A x^{\prime}(t)+M(t) x(t)+N(t) \max _{s \in[t-h, t]} x(s) \leq 0, \quad t \in[0, T] \\
x(0) \leq x(T), \quad x(t)=x(0), \quad t \in[-h, 0]
\end{array}\right.
$$

where $A, M(t)$ are $n \times n$ matrices, $A$ is singular and $M(t), N(t)$ are continuous on $[0, T]$.

Lemma 2.3 Assume that the conditions $\left(\mathrm{H}_{2.1}\right)-\left(\mathrm{H}_{2.4}\right)$ hold, and

$\left(\mathrm{H}_{2.5}\right)$ there exists a matrix $N$ such that $N \leq N(t) \leq 0$ on $[0, T]$, and the matrix $[I-M]^{-1}$ exists and is nonnegative, where

$$
M=\max _{s \in[0, T]}\left\{-[\lambda A+M(s)]^{-1}\left[\hat{A} \hat{A}^{D} \int_{0}^{T} G(t, \sigma) N d \sigma+\left(I-\hat{A} \hat{A}^{D}\right) \hat{M}^{D} N\right]\right\} .
$$

Then $x(t) \leq 0$ on $[-h, T]$.

Proof In view of the condition $\left(\mathrm{H}_{2.5}\right)$, we can get from (2.7)

$$
\begin{aligned}
& A x^{\prime}(t)+M(t) x(t) \leq-N(t) \max _{s \in[-h, T]} x(s)=-N(t) \max _{s \in[0, T]} x(s) \\
& \leq-N \max _{s \in[0, T]} x(s), \quad t \in[0, T], \\
& x(0) \leq x(T), \quad x(t)=x(0), \quad t \in[-h, 0] .
\end{aligned}
$$

Lemma 2.1 shows that $x(t) \leq y(t)$ on $[0, T]$, where $y(t)$ is the solution of

$$
\begin{aligned}
& A y^{\prime}(t)+M(t) y(t)=-N \max _{s \in[0, T]} x(s), \quad t \in[0, T], \\
& y(0)=y(T), \quad y(t)=y(0), \quad t \in[-h, 0] .
\end{aligned}
$$


Thus, for $t \in[0, T]$, using the expression of $x(t)$ in Lemma 2.2, we obtain

$$
\begin{aligned}
y(t)= & -[\lambda A+M(t)]^{-1}\left[\hat{A} \hat{A}^{D} \int_{0}^{T} G(t, \sigma) N \max _{s \in[0, T]} x(s) d \sigma\right. \\
& \left.+\left(I-\hat{A} \hat{A}^{D}\right) \hat{M}^{D} N \max _{s \in[0, T]} x(s)\right] .
\end{aligned}
$$

Hence, we have

$$
\begin{aligned}
\max _{s \in[0, T]} x(s) \leq & \max _{s \in[0, T]}\left\{-[\lambda A+M(s)]^{-1}\left[\hat{A} \hat{A}^{D} \int_{0}^{T} G(t, \sigma) N d \sigma\right.\right. \\
& \left.\left.+\left(I-\hat{A} \hat{A}^{D}\right) \hat{M}^{D} N\right]\right\} \max _{s \in[0, T]} x(s) \\
= & M \max _{s \in[0, T]} x(s),
\end{aligned}
$$

using the condition $\left(\mathrm{H}_{2.5}\right)$, we have $\max _{s \in[0, T]} x(s) \leq 0$. Then we obtain $x(t) \leq 0$ on $[0, T]$. Furthermore, we conclude that $x(t) \leq 0$ on $[-h, T]$. The proof is complete.

Next, we will prove an existence result for the PBVP (2.1), which is vital for our main results.

Lemma 2.4 Assume that the conditions $\left(\mathrm{H}_{2.1}\right)-\left(\mathrm{H}_{2.5}\right)$ hold, and

$\left(\mathrm{H}_{2.6}\right)$ the functions $\alpha_{0}, \beta_{0} \in C\left([-h, T], R^{n}\right) \cup C^{1}\left([0, T], R^{n}\right)$ are lower and upper solutions of $\operatorname{PBVP}(2.1)$, and $\alpha_{0}(t) \leq \beta_{0}(t)$ on $[-h, T] ;$

$\left(\mathrm{H}_{2.7}\right)$ there exists a function $f \in C\left(\Omega\left(\alpha_{0}, \beta_{0}\right), R^{n}\right)$ that satisfies the inequality

$$
f(t, y, v)-f(t, x, u) \leq-M_{0}(y-x)-N_{0}(v-u)
$$

where $y \leq x, v \leq u, M\left(t_{0}\right)=M_{0}, N\left(t_{0}\right)=N_{0}, t_{0} \in[0, T]$.

Then there exists a solution $x$ of PBVP (2.1) such that $\alpha_{0}(t) \leq x(t) \leq \beta_{0}(t)$ on $[-h, T]$.

Proof Consider the iterative scheme

$$
\left\{\begin{array}{l}
A U_{n+1}^{\prime}(t)=Q_{n+1}(t)-M_{0} U_{n+1}(t)-N_{0} \max _{s \in[t-h, t]} U_{n+1}(s), \quad t \in[0, T] \\
U_{n+1}(0)=U_{n+1}(T), \quad U_{n+1}(t)=U_{n+1}(0), \quad t \in[-h, 0]
\end{array}\right.
$$

where $Q_{n+1}(t)=f\left(t, U_{n}(t), \max _{s \in[t-h, t]} U_{n}(s)\right)+M_{0} U_{n}(t)+N_{0} \max _{s \in[t-h, t]} U_{n}(s)$. According to the iterative scheme, the sequences $\left\{\alpha_{n}(t)\right\}$ and $\left\{\beta_{n}(t)\right\}$ were generated by $\alpha_{0}(t)$ and $\beta_{0}(t)$, respectively.

Now, we will prove that

$$
\alpha_{0}(t) \leq \alpha_{1}(t) \leq \beta_{1}(t) \leq \beta_{0}(t), \quad t \in[-h, T]
$$

For this purpose, setting $p_{1}(t)=\alpha_{0}(t)-\alpha_{1}(t)$, using the condition $\left(\mathrm{H}_{2.6}\right)$, we have

$$
\begin{aligned}
& A p_{1}^{\prime}(t) \leq-M_{0} p_{1}(t)-N_{0} \max _{s \in[t-h, t]} p_{1}(s), \quad t \in[0, T] \\
& p_{1}(0) \leq p_{1}(T), \quad p_{1}(t)=p_{1}(0), \quad t \in[-h, 0] .
\end{aligned}
$$


According to Lemma 2.3, we have $p_{1}(t) \leq 0$ on $[-h, T]$. Similarly, setting $p_{2}(t)=\beta_{1}(t)-$ $\beta_{0}(t)$, we can obtain $p_{2}(t) \leq 0$ on $[-h, T]$.

Letting $p_{3}(t)=\alpha_{1}(t)-\beta_{1}(t)$, from the condition $\left(\mathrm{H}_{2.7}\right)$, we have

$$
\begin{aligned}
& A p_{3}^{\prime}(t) \leq-M_{0} p_{3}(t)-N_{0} \max _{s \in[t-h, t]} p_{3}(s), \quad t \in[0, T], \\
& p_{3}(0)=p_{3}(T), \quad p_{3}(t)=p_{3}(0), \quad t \in[-h, 0] .
\end{aligned}
$$

By Lemma 2.3, we have $p_{3}(t) \leq 0$ on $[-h, T]$.

The process can be continued to obtain

$$
\alpha_{0}(t) \leq \alpha_{1}(t) \leq \cdots \leq \alpha_{n}(t) \leq \beta_{n}(t) \leq \cdots \leq \beta_{1}(t) \leq \beta_{0}(t), \quad t \in[-h, T]
$$

It is easy to see that the sequence $\left\{\alpha_{n}(t)\right\}$ is uniformly bounded and equicontinuous, employing the Ascoli-Arzela theorem, the nondecreasing sequence $\left\{\alpha_{n}(t)\right\}$ converges pointwise to a function $x(t)$ that satisfies $\alpha_{0}(t) \leq x(t) \leq \beta_{0}(t)$. In view of PBVP (2.8) and the Dominated Convergence Theorem, we see that $x(t)$ is a solution of

$$
\begin{aligned}
& A x^{\prime}(t)=f\left(t, x(t), \max _{s \in[t-h, t]} x(s)\right)-M_{0}(x(t)-x(t))-N_{0}(x(t)-x(t)), \quad t \in[0, T], \\
& x(0)=x(T), \quad x(t)=x(0), \quad t \in[-h, 0],
\end{aligned}
$$

that is, $x(t)$ is a solution of PBVP (2.1). Therefore, we conclude that there exists a solution $x(t)$ of PBVP $(2.1)$ that satisfies $\alpha_{0}(t) \leq x(t) \leq \beta_{0}(t)$ on $[-h, T]$. The proof is complete.

\section{Quasilinearization technique}

We apply the method of quasilinearization to nonlinear singular differential system with 'maxima'. By assuming suitable conditions on the function $f$, we see that there exist two monotone sequences which converge quadratically to the solution of PBVP (2.1).

Theorem 3.1 Assume that the conditions $\left(\mathrm{H}_{2.1}\right)-\left(\mathrm{H}_{2.7}\right)$ hold, and

$\left(\mathrm{A}_{3.1}\right)$ there exists a function $f \in C^{0,2,2}\left(\Omega\left(\alpha_{0}, \beta_{0}\right), R^{n}\right)$ such that $f_{y} \geq 0$ and $H(f) \geq 0$, where

$$
\begin{aligned}
& H(f)= \int_{0}^{1}\left[(x(t)-y(t)) \frac{\partial}{\partial x}+\left(\max _{s \in[t-h, t]} x(s)-\max _{s \in[t-h, t]} y(s)\right) \frac{\partial}{\partial y}\right]^{2} \\
& \times f\left(t, \sigma x(t)+(1-\sigma) y(t), \sigma \max _{s \in[t-h, t]} x(s)+(1-\sigma) \max _{s \in[t-h, t]} y(s)\right) d \sigma \\
& \text { for } \alpha_{0}(t) \leq x(t), y(t) \leq \beta_{0}(t) \text { on }[-h, T] .
\end{aligned}
$$

Then there exist two sequences $\left\{\alpha_{n}(t)\right\}$ and $\left\{\beta_{n}(t)\right\}$ converging uniformly to the solution $x(t)$ of PBVP (2.1) and the convergence is quadratic, that is, there exist positive matrices $K_{1}, K_{2}$ such that the solution $x(t)$ of PBVP $(2.1)$ in $S\left(\alpha_{0}, \beta_{0}\right)$, the inequalities

$$
\begin{aligned}
& \left|x-\alpha_{n+1}\right|_{0} \leq K_{1}\left|x-\alpha_{n}\right|_{0}^{2}, \\
& \left|\beta_{n+1}-x\right|_{0} \leq K_{2}\left|\beta_{n}-x\right|_{0}^{2}
\end{aligned}
$$


hold, where

$$
|u|_{0}=\max _{s \in[-h, T]}|u(s)|=\left(\max _{s \in[-h, T]}\left|u_{1}(s)\right|, \max _{s \in[-h, T]}\left|u_{2}(s)\right|, \ldots, \max _{s \in[-h, T]}\left|u_{n}(s)\right|\right)^{T}
$$

Proof From the condition $\left(\mathrm{A}_{3.1}\right)$, we obtain

$$
\begin{aligned}
f\left(t, x(t), \max _{s \in[t-h, t]} x(s)\right) \geq & f\left(t, y(t), \max _{s \in[t-h, t]} y(s)\right)+f_{x}\left(t, y(t), \max _{s \in[t-h, t]} y(s)\right)(x(t)-y(t)) \\
& +f_{y}\left(t, y(t), \max _{s \in[t-h, t]} y(s)\right)\left(\max _{s \in[t-h, t]} x(s)-\max _{s \in[t-h, t]} y(s)\right)
\end{aligned}
$$

and

$$
\begin{aligned}
f\left(t, x(t), \max _{s \in[t-h, t]} x(s)\right) \leq & f\left(t, y(t), \max _{s \in[t-h, t]} y(s)\right)+f_{x}\left(t, y(t), \max _{s \in[t-h, t]} y(s)\right)(x(t)-y(t)) \\
& +f_{y}\left(t, y(t), \max _{s \in[t-h, t]} y(s)\right)\left(\max _{s \in[t-h, t]} x(s)-\max _{s \in[t-h, t]} y(s)\right) \\
& +\frac{1}{2} M_{1}\left(|x(t)-y(t)|^{2}+\left|\max _{s \in[t-h, t]} x(s)-\max _{s \in[t-h, t]} y(s)\right|^{2}\right),
\end{aligned}
$$

where

$$
\begin{aligned}
H(f) \leq & \left(\sum_{j=1}^{n}\left|f_{x_{j} x}\right|+\sum_{j=1}^{n}\left|f_{y_{j} x}\right|\right)|x(t)-y(t)|^{2} \\
& +\left(\sum_{j=1}^{n}\left|f_{x_{j} y}\right|+\sum_{j=1}^{n}\left|f_{y_{j} y}\right|\right)\left|\max _{s \in[t-h, t]} x(s)-\max _{s \in[t-h, t]} y(s)\right|^{2} \\
\leq & \left(M_{11}+M_{13}\right)|x(t)-y(t)|^{2}+\left(M_{12}+M_{14}\right)\left|\max _{s \in[t-h, t]} x(s)-\max _{s \in[t-h, t]} y(s)\right|^{2} \\
\leq & M_{1}\left(|x(t)-y(t)|^{2}+\left|\max _{s \in[t-h, t]} x(s)-\max _{s \in[t-h, t]} y(s)\right|^{2}\right)
\end{aligned}
$$

with $\alpha_{0}(t) \leq x(t), y(t) \leq \beta_{0}(t), \sum_{j=1}^{n}\left|f_{x_{j} x}\right| \leq M_{11}, \quad \sum_{j=1}^{n}\left|f_{x_{j} y}\right| \leq M_{12}, \quad \sum_{j=1}^{n}\left|f_{y_{j}}\right| \leq M_{13}$, $\sum_{j=1}^{n}\left|f_{y_{j}}\right| \leq M_{14}$ for $t \in[0, T], M_{11}, M_{12}, M_{13}$, and $M_{14}$ are positive matrices, and $M_{1}=$ $M_{11}+M_{12}+M_{13}+M_{14}$.

Now, we consider the singular differential system with 'maxima'

$$
\left\{\begin{aligned}
A x^{\prime}(t)= & f\left(t, \alpha_{0}(t), \max _{s \in[t-h, t]} \alpha_{0}(s)\right)+f_{x}\left(t, \alpha_{0}(t), \max _{s \in[t-h, t]} \alpha_{0}(s)\right)\left(x(t)-\alpha_{0}(t)\right) \\
& +f_{y}\left(t, \alpha_{0}(t), \max _{s \in[t-h, t]} \alpha_{0}(s)\right)\left(\max _{s \in[t-h, t]} x(s)-\max _{s \in[t-h, t]} \alpha_{0}(s)\right) \\
\equiv & F_{0}\left(t, x(t), \max _{s \in[t-h, t]} x(s)\right), \quad t \in[0, T], \\
x(0)= & x(T), \quad x(t)=x(0), \quad t \in[-h, 0] .
\end{aligned}\right.
$$

We shall now show that $\alpha_{0}(t)$ and $\beta_{0}(t)$ are lower and upper solutions of PBVP (3.3), respectively. In fact, the condition $\left(\mathrm{H}_{2.6}\right)$ and inequality (3.1) imply

$$
\begin{aligned}
& A \alpha_{0}^{\prime}(t) \leq f\left(t, \alpha_{0}(t), \max _{s \in[t-h, t]} \alpha_{0}(s)\right)=F_{0}\left(t, \alpha_{0}(t), \max _{s \in[t-h, t]} \alpha_{0}(s)\right), \quad t \in[0, T], \\
& \alpha_{0}(0) \leq \alpha_{0}(T), \quad \alpha_{0}(t)=\alpha_{0}(0), \quad t \in[-h, 0]
\end{aligned}
$$


and

$$
\begin{aligned}
A \beta_{0}^{\prime}(t) \geq & f\left(t, \alpha_{0}(t), \max _{s \in[t-h, t]} \alpha_{0}(s)\right)+f_{x}\left(t, \alpha_{0}(t), \max _{s \in[t-h, t]} \alpha_{0}(s)\right)\left(\beta_{0}(t)-\alpha_{0}(t)\right) \\
& +f_{y}\left(t, \alpha_{0}(t), \max _{s \in[t-h, t]} \alpha_{0}(s)\right)\left(\max _{s \in[t-h, t]} \beta_{0}(s)-\max _{s \in[t-h, t]} \alpha_{0}(s)\right) \\
= & F_{0}\left(t, \beta_{0}(t), \max _{s \in[t-h, t]} \beta_{0}(s)\right), \quad t \in[0, T], \\
\beta_{0}(0) \geq & \beta_{0}(T), \quad \beta_{0}(t)=\beta_{0}(0), \quad t \in[-h, 0] .
\end{aligned}
$$

Hence, by Lemma 2.4, there exists a solution $\alpha_{1}(t)$ of PBVP (3.3) such that $\alpha_{0}(t) \leq \alpha_{1}(t) \leq$ $\beta_{0}(t)$ on $[-h, T]$.

Similarly, consider the singular differential system with 'maxima'

$$
\left\{\begin{aligned}
A x^{\prime}(t)= & f\left(t, \beta_{0}(t), \max _{s \in[t-h, t]} \beta_{0}(s)\right)+f_{x}\left(t, \beta_{0}(t), \max _{s \in[t-h, t]} \beta_{0}(s)\right)\left(x(t)-\beta_{0}(t)\right) \\
& +f_{y}\left(t, \beta_{0}(t), \max _{s \in[t-h, t]} \beta_{0}(s)\right)\left(\max _{s \in[t-h, t]} x(s)-\max _{s \in[t-h, t]} \beta_{0}(s)\right) \\
& +\frac{1}{2} M_{1}\left(\left|x(t)-\beta_{0}(t)\right|^{2}+\left|\max _{s \in[t-h, t]} x(s)-\max _{s \in[t-h, t]} \beta_{0}(s)\right|^{2}\right) \\
\equiv & G_{0}\left(t, x(t), \max _{s \in[t-h, t]} x(s)\right), \quad t \in[0, T], \\
x(0)= & x(T), \quad x(t)=x(0), \quad t \in[-h, 0] .
\end{aligned}\right.
$$

We show that $\alpha_{1}(t)$ and $\beta_{0}(t)$ are lower and upper solutions of PBVP (3.4), respectively. Using the conclusion that $\alpha_{1}(t)$ is a solution of PBVP (3.3), the condition $\left(\mathrm{H}_{2.6}\right)$, and inequalities (3.1), (3.2), we obtain

$$
\begin{aligned}
A \alpha_{1}^{\prime}(t)= & f\left(t, \alpha_{0}(t), \max _{s \in[t-h, t]} \alpha_{0}(s)\right)+f_{x}\left(t, \alpha_{0}(t), \max _{s \in[t-h, t]} \alpha_{0}(s)\right)\left(\alpha_{1}(t)-\alpha_{0}(t)\right) \\
& +f_{y}\left(t, \alpha_{0}(t), \max _{s \in[t-h, t]} \alpha_{0}(s)\right)\left(\max _{s \in[t-h, t]} \alpha_{1}(s)-\max _{s \in[t-h, t]} \alpha_{0}(s)\right) \\
\leq & f\left(t, \alpha_{1}(t) \max _{s \in[t-h, t]} \alpha_{1}(s)\right) \\
\leq & f\left(t, \beta_{0}(t), \max _{s \in[t-h, t]} \beta_{0}(s)\right)+f_{x}\left(t, \beta_{0}(t), \max _{s \in[t-h, t]} \beta_{0}(s)\right)\left(\alpha_{1}(t)-\beta_{0}(t)\right) \\
& +f_{y}\left(t, \beta_{0}(t) \max _{s \in[t-h, t]} \beta_{0}(s)\right)\left(\max _{s \in[t-h, t]} \alpha_{1}(s)-\max _{s \in[t-h, t]} \beta_{0}(s)\right) \\
& +\frac{1}{2} M_{1}\left(\left|\alpha_{1}(t)-\beta_{0}(t)\right|^{2}+\left|\max _{s \in[t-h, t]} \alpha_{1}(s)-\max _{s \in[t-h, t]} \beta_{0}(s)\right|^{2}\right) \\
\equiv & G_{0}\left(t, \alpha_{1}(t), \max _{s \in[t-h, t]} \alpha_{1}(s)\right), \quad t \in[0, T], \\
\alpha_{1}(0)= & \alpha_{1}(T), \quad \alpha_{1}(t)=\alpha_{1}(0), \quad t \in[-h, 0]
\end{aligned}
$$

and

$$
\begin{aligned}
& A \beta_{0}^{\prime}(t) \geq f\left(t, \beta_{0}(t), \max _{s \in[t-h, t]} \beta_{0}(s)\right)=G_{0}\left(t, \beta_{0}(t), \max _{s \in[t-h, t]} \beta_{0}(s)\right), \quad t \in[0, T], \\
& \beta_{0}(0) \geq \beta_{0}(T), \quad \beta_{0}(t)=\beta_{0}(0), \quad t \in[-h, 0] .
\end{aligned}
$$

These imply that $\alpha_{1}(t)$ and $\beta_{0}(t)$ are lower and upper solutions of PBVP (3.4), respectively. Thus, as before, there exists a solution $\beta_{1}(t)$ of PBVP (3.4) such that $\alpha_{1}(t) \leq \beta_{1}(t) \leq \beta_{0}(t)$ on $[-h, T]$. 
Next, we will show that $\alpha_{1}(t)$ and $\beta_{1}(t)$ are lower and upper solutions of PBVP (2.1), respectively. Since $\alpha_{1}(t)$ is a solution of PBVP (3.3), using the inequality (3.1), we get

$$
\begin{aligned}
A \alpha_{1}^{\prime}(t)= & f\left(t, \alpha_{0}(t), \max _{s \in[t-h, t]} \alpha_{0}(s)\right)+f_{x}\left(t, \alpha_{0}(t), \max _{s \in[t-h, t]} \alpha_{0}(s)\right)\left(\alpha_{1}(t)-\alpha_{0}(t)\right) \\
& +f_{y}\left(t, \alpha_{0}(t), \max _{s \in[t-h, t]} \alpha_{0}(s)\right)\left(\max _{s \in[t-h, t]} \alpha_{1}(s)-\max _{s \in[t-h, t]} \alpha_{0}(s)\right) \\
\leq & f\left(t, \alpha_{1}(t), \max _{s \in[t-h, t]} \alpha_{1}(s)\right), \quad t \in[0, T] \\
\alpha_{1}(0)= & \alpha_{1}(T), \quad \alpha_{1}(t)=\alpha_{1}(0), \quad t \in[-h, 0] .
\end{aligned}
$$

This shows that $\alpha_{1}(t)$ is a lower solution of PBVP (2.1) on $[-h, T]$. Similarly, since $\beta_{1}(t)$ is a solution of PBVP (3.4), it follows from the inequality (3.2) that

$$
\begin{aligned}
A \beta_{1}^{\prime}(t)= & f\left(t, \beta_{0}(t), \max _{s \in[t-h, t]} \beta_{0}(s)\right)+f_{x}\left(t, \beta_{0}(t), \max _{s \in[t-h, t]} \beta_{0}(s)\right)\left(\beta_{1}(t)-\beta_{0}(t)\right) \\
& +f_{y}\left(t, \beta_{0}(t), \max _{s \in[t-h, t]} \beta_{0}(s)\right)\left(\max _{s \in[t-h, t]} \beta_{1}(s)-\max _{s \in[t-h, t]} \beta_{0}(s)\right) \\
& +\frac{1}{2} M_{1}\left(\left|\beta_{1}(t)-\beta_{0}(t)\right|^{2}+\left|\max _{s \in[t-h, t]} \beta_{1}(s)-\max _{s \in[t-h, t]} \beta_{0}(s)\right|^{2}\right) \\
\geq & f\left(t, \beta_{1}(t), \max _{s \in[t-h, t]} \beta_{1}(s)\right), \quad t \in[0, T], \\
\beta_{1}(0)= & \beta_{1}(T), \quad \beta_{1}(t)=\beta_{1}(0), \quad t \in[-h, 0] .
\end{aligned}
$$

This proves that $\beta_{1}(t)$ is an upper solution of $\operatorname{PBVP}(2.1)$ on $[-h, T]$. As a result, we get

$$
\alpha_{0}(t) \leq \alpha_{1}(t) \leq \beta_{1}(t) \leq \beta_{0}(t), \quad t \in[-h, T]
$$

Continuing this process by induction, we obtain

$$
\alpha_{n}(t) \leq \alpha_{n+1}(t) \leq \beta_{n+1}(t) \leq \beta_{n}(t), \quad t \in[-h, T]
$$

where $\alpha_{n+1}(t)$ and $\beta_{n+1}(t)$ are solutions of the singular differential systems with 'maxima'

$$
\left\{\begin{aligned}
& A x^{\prime}(t)= f\left(t, \alpha_{n}(t), \max _{s \in[t-h, t]} \alpha_{n}(s)\right)+f_{x}\left(t, \alpha_{n}(t), \max _{s \in[t-h, t]} \alpha_{n}(s)\right)\left(x(t)-\alpha_{n}(t)\right) \\
&+f_{y}\left(t, \alpha_{n}(t), \max _{s \in[t-h, t]} \alpha_{n}(s)\right)\left(\max _{s \in[t-h, t]} x(s)-\max _{s \in[t-h, t]} \alpha_{n}(s)\right) \\
& \equiv F_{n}\left(t, x(t), \max _{s \in[t-h, t]} x(s)\right), \quad t \in[0, T], \\
& x(0)=x(T), \quad x(t)=x(0), \quad t \in[-h, 0]
\end{aligned}\right.
$$

and

$$
\left\{\begin{aligned}
A x^{\prime}(t)= & f\left(t, \beta_{n}(t), \max _{s \in[t-h, t]} \beta_{n}(s)\right)+f_{x}\left(t, \beta_{n}(t), \max _{s \in[t-h, t]} \beta_{n}(s)\right)\left(x(t)-\beta_{n}(t)\right) \\
& +f_{y}\left(t, \beta_{n}(t), \max _{s \in[t-h, t]} \beta_{n}(s)\right)\left(\max _{s \in[t-h, t]} x(s)-\max _{s \in[t-h, t]} \beta_{n}(s)\right) \\
& +\frac{1}{2} M_{1}\left(\left|x(t)-\beta_{n}(t)\right|^{2}+\left|\max _{s \in[t-h, t]} x(s)-\max _{s \in[t-h, t]} \beta_{n}(s)\right|^{2}\right) \\
\equiv & G_{n}\left(t, x(t), \max _{s \in[t-h, t]} x(s)\right), \quad t \in[0, T], \\
x(0)= & x(T), \quad x(t)=x(0), \quad t \in[-h, 0] .
\end{aligned}\right.
$$


By induction we have, for all $n$,

$$
\alpha_{0}(t) \leq \alpha_{1}(t) \leq \cdots \leq \alpha_{n}(t) \leq \beta_{n}(t) \leq \cdots \leq \beta_{1}(t) \leq \beta_{0}(t), \quad t \in[-h, T] .
$$

Clearly, the sequences $\left\{\alpha_{n}(t)\right\},\left\{\beta_{n}(t)\right\}$ are equicontinuous and uniformly bounded. Thus, employing the Ascoli-Arzela theorem, we see that the monotone sequences $\left\{\alpha_{n}(t)\right\}$ and $\left\{\beta_{n}(t)\right\}$ converge uniformly and monotonically on $[-h, T]$ with

$$
\begin{aligned}
& \lim _{n \rightarrow \infty} \alpha_{n}(t)=\rho(t), \\
& \lim _{n \rightarrow \infty} \beta_{n}(t)=r(t) .
\end{aligned}
$$

We shall prove that the convergence of $\left\{\alpha_{n}(t)\right\}$ and $\left\{\beta_{n}(t)\right\}$ is quadratic. To do this, let $x(t)$ be a solution of PBVP $(2.1)$ in $S\left(\alpha_{0}, \beta_{0}\right)$. Define

$$
a_{n+1}(t)=x(t)-\alpha_{n+1}(t) \geq 0, \quad t \in[-h, T] .
$$

Case 1. $t \in[-h, 0], a_{n+1}(t)=a_{n+1}(0)=a_{n+1}(T)$.

Case 2. $t \in[0, T]$. In view of the assumption $f_{y} \geq 0$ and the inequality (3.2), we have

$$
\begin{aligned}
A a_{n+1}^{\prime}(t)= & f\left(t, x(t), \max _{s \in[t-h, t]} x(s)\right)-\left[f\left(t, \alpha_{n}(t), \max _{s \in[t-h, t]} \alpha_{n}(s)\right)\right. \\
& +f_{x}\left(t, \alpha_{n}(t), \max _{s \in[t-h, t]} \alpha_{n}(s)\right)\left(\alpha_{n+1}(t)-\alpha_{n}(t)\right) \\
& \left.+f_{y}\left(t, \alpha_{n}(t), \max _{s \in[t-h, t]} \alpha_{n}(s)\right)\left(\max _{s \in[t-h, t]} \alpha_{n+1}(s)-\max _{s \in[t-h, t]} \alpha_{n}(s)\right)\right] \\
\leq & f_{x}\left(t, \alpha_{n}(t), \max _{s \in[t-h, t]} \alpha_{n}(s)\right)\left(x(t)-\alpha_{n}(t)\right) \\
& +f_{y}\left(t, \alpha_{n}(t), \max _{s \in[t-h, t]} \alpha_{n}(s)\right)\left(\max _{s \in[t-h, t]} x(s)-\max _{s \in[t-h, t]} \alpha_{n}(s)\right) \\
& +\frac{1}{2} M_{1}\left(\left|x(t)-\alpha_{n}(t)\right|^{2}+\max _{s \in[t-h, t]}\left|x(s)-\alpha_{n}(s)\right|^{2}\right) \\
& -f_{x}\left(t, \alpha_{n}(t), \max _{s \in[t-h, t]} \alpha_{n}(s)\right)\left(\alpha_{n+1}(t)-\alpha_{n}(t)\right) \\
& -f_{y}\left(t, \alpha_{n}(t), \max _{s \in[t-h, t]} \alpha_{n}(s)\right)\left(\max _{s \in[t-h, t]} \alpha_{n+1}(s)-\max _{s \in[t-h, t]} \alpha_{n}(s)\right) \\
\leq & f_{x}\left(t, \alpha_{n}(t), \max _{s \in[t-h, t]} \alpha_{n}(s)\right)\left(x(t)-\alpha_{n+1}(t)\right) \\
& +f_{y}\left(t, \alpha_{n}(t), \max _{s \in[t-h, t]} \alpha_{n}(s)\right) \max _{s \in[t-h, t]}\left(x(s)-\alpha_{n+1}(s)\right) \\
& +\frac{1}{2} M_{1}\left(\left|x(t)-\alpha_{n}(t)\right|^{2}+\left|\max _{s \in[t-h, t]} x(s)-\max _{s \in[t-h, t]} \alpha_{n}(s)\right|^{2}\right) \\
\leq & M(\bar{t}) a_{n+1}(t)-N(\bar{t}) \max _{s \in[t-h, t]} a_{n+1}(s)+M_{1} \max _{s \in[t-h, t]}\left|a_{n}(s)\right|^{2} \\
\leq & M(\bar{t}) a_{n+1}(t)-N(\bar{t}) \max _{s \in[-h, T]} a_{n+1}(s)+M_{1} \max _{s \in[-h, T]}\left|a_{n}(s)\right|^{2} \\
= & M(\bar{t}) a_{n+1}(t)-N(\bar{t}) \max _{s \in[0, T]} a_{n+1}(s)+M_{1} \max _{s \in[0, T]}\left|a_{n}(s)\right|^{2}, \\
& \\
& \\
& \\
&
\end{aligned}
$$


where $f_{x}(t, x, y) \leq-M(\bar{t}), f_{y}(t, x, y) \leq-N(\bar{t})$ for $(t, x, y) \in \Omega\left(\alpha_{0}, \beta_{0}\right), \bar{t} \in[0, T]$. Then we obtain

$$
\begin{aligned}
& A a_{n+1}^{\prime}(t) \leq-M(\bar{t}) a_{n+1}(t)-N(\bar{t}) \max _{s \in[0, T]} a_{n+1}(s)+M_{1} \max _{s \in[0, T]}\left|a_{n}(s)\right|^{2}, \quad t \in[0, T], \\
& a_{n+1}(0)=a_{n+1}(T), \quad a_{n+1}(t)=a_{n+1}(0), \quad t \in[-h, 0] .
\end{aligned}
$$

According to Lemma 2.1, we see that $a_{n+1}(t) \leq u(t)$ on $[0, T]$, where $u(t)$ is the solution of

$$
\begin{aligned}
& A u^{\prime}+M(\bar{t}) u=-N(\bar{t}) \max _{s \in[0, T]} a_{n+1}(s)+M_{1} \max _{s \in[0, T]}\left|a_{n}(s)\right|^{2}, \quad t \in[0, T], \\
& u(0)=u(T), \quad u(t)=u(0), \quad t \in[-h, 0] .
\end{aligned}
$$

By using the expression of $x(t)$ in Lemma 2.2 and taking suitable estimates, we conclude that

$$
\left|a_{n+1}\right|_{0} \leq K_{1}\left|a_{n}\right|_{0}^{2}
$$

where $K_{1}$ is a positive matrix. This shows that the convergence of $\left\{\alpha_{n}(t)\right\}$ is quadratic.

Similarly, consider

$$
b_{n+1}(t)=\beta_{n+1}(t)-x(t) \geq 0, \quad t \in[-h, T] .
$$

Case 1. If $t \in[-h, 0], b_{n+1}(t)=b_{n+1}(0)=b_{n+1}(T)$.

Case 2. If $t \in[0, T]$. The assumption $f_{y} \geq 0$ and the inequality (3.1) yield

$$
\begin{aligned}
A b_{n+1}^{\prime}(t)= & f\left(t, \beta_{n}(t), \max _{s \in[t-h, t]} \beta_{n}(s)\right)+f_{x}\left(t, \beta_{n}(t), \max _{s \in[t-h, t]} \beta_{n}(s)\right)\left(\beta_{n+1}(t)-\beta_{n}(t)\right) \\
& +f_{y}\left(t, \beta_{n}(t), \max _{s \in[t-h, t]} \beta_{n}(s)\right)\left(\max _{s \in[t-h, t]} \beta_{n+1}(s)-\max _{s \in[t-h, t]} \beta_{n}(s)\right) \\
& +\frac{1}{2} M_{1}\left(\left|\beta_{n+1}(t)-\beta_{n}(t)\right|^{2}+\left|\max _{s \in[t-h, t]} \beta_{n+1}(s)-\max _{s \in[t-h, t]} \beta_{n}(s)\right|^{2}\right) \\
& -f\left(t, x(t) \max _{s \in[t-h, t]} x(s)\right) \\
\leq & f\left(t, \beta_{n}(t), \max _{s \in[t-h, t]} \beta_{n}(s)\right)+f_{x}\left(t, \beta_{n}(t), \max _{s \in[t-h, t]} \beta_{n}(s)\right)\left(\beta_{n+1}(t)-\beta_{n}(t)\right) \\
& +f_{y}\left(t, \beta_{n}(t), \max _{s \in[t-h, t]} \beta_{n}(s)\right)\left(\max _{s \in[t-h, t]} \beta_{n+1}(s)-\max _{s \in[t-h, t]} \beta_{n}(s)\right) \\
& +\frac{1}{2} M_{1}\left(\left|\beta_{n+1}(t)-\beta_{n}(t)\right|^{2}+\left|\max _{s \in[t-h, t]} \beta_{n+1}(s)-\max _{s \in[t-h, t]} \beta_{n}(s)\right|^{2}\right) \\
& -\left[f\left(t, \beta_{n}(t), \max _{s \in[t-h, t]} \beta_{n}(s)\right)+f_{x}\left(t, \beta_{n}(t), \max _{s \in[t-h, t]} \beta_{n}(s)\right)\left(x(t)-\beta_{n}(t)\right)\right. \\
& \left.+f_{y}\left(t, \beta_{n}(t), \max _{s \in[t-h, t]} \beta_{n}(s)\right)\left(\max _{s \in[t-h, t]} x(s)-\max _{s \in[t-h, t]} \beta_{n}(s)\right)\right] \\
\leq & f_{x}\left(t, \beta_{n}(t), \max _{s \in[t-h, t]} \beta_{n}(s)\right)\left(\beta_{n+1}(t)-x(t)\right) \\
& +f_{y}\left(t, \beta_{n}(t), \max _{s \in[t-h, t]} \beta_{n}(s)\right) \max _{s \in[t-h, t]}\left(\beta_{n+1}(s)-x(s)\right)
\end{aligned}
$$




$$
\begin{aligned}
& +\frac{1}{2} M_{1}\left(\left|x(t)-\beta_{n}(t)\right|^{2}+\left|\max _{s \in[t-h, t]} x(s)-\max _{s \in[t-h, t]} \beta_{n}(s)\right|^{2}\right) \\
\leq & -M(\bar{t}) b_{n+1}(t)-N(\bar{t}) \max _{s \in[t-h, t]} b_{n+1}(s)+M_{1} \max _{s \in[t-h, t]}\left|b_{n}(s)\right|^{2} \\
\leq & -M(\bar{t}) b_{n+1}(t)-N(\bar{t}) \max _{s \in[0, T]} b_{n+1}(s)+M_{1} \max _{s \in[0, T]}\left|b_{n}(s)\right|^{2} .
\end{aligned}
$$

Then we have

$$
\begin{aligned}
& A b_{n+1}^{\prime}(t) \leq-M(\bar{t}) b_{n+1}(t)-N(\bar{t}) \max _{s \in[0, T]} b_{n+1}(s)+M_{1} \max _{s \in[0, T]}\left|b_{n}(s)\right|^{2}, \quad t \in[0, T], \\
& b_{n+1}(0)=b_{n+1}(T), \quad b_{n+1}(t)=b_{n+1}(0), \quad t \in[-h, 0] .
\end{aligned}
$$

By Lemma 2.1, we see that $b_{n+1}(t) \leq u(t)$ on $[0, T]$, where $u(t)$ is the solution of

$$
\begin{aligned}
& A u^{\prime}+M(\bar{t}) u=-N(\bar{t}) \max _{s \in[0, T]} b_{n+1}(s)+M_{1} \max _{s \in[0, T]}\left|b_{n}(s)\right|^{2}, \quad t \in[0, T], \\
& u(0)=u(T), \quad u(t)=u(0), \quad t \in[-h, 0] .
\end{aligned}
$$

Hence, applying the expression of $x(t)$ in Lemma 2.2 and taking suitable estimates, we obtain

$$
\left|b_{n+1}\right|_{0} \leq K_{2}\left|b_{n}\right|_{0}^{2}
$$

where $K_{2}$ is a positive matrix. Thus, the convergence of $\left\{\beta_{n}(t)\right\}$ is quadratic. The proof is complete.

\section{Generalized quasilinearization technique}

We apply the method of generalized quasilinearization to nonlinear singular differential system with 'maxima'. By assuming less restrictive conditions on the function $f$, we see that there exist two monotone sequences which converge quadratically to the solution of $\operatorname{PBVP}(2.1)$.

Theorem 4.1 Assume that the conditions $\left(\mathrm{H}_{2.1}\right)-\left(\mathrm{H}_{2.7}\right)$ hold, and

$\left(\mathrm{A}_{4.1}\right)$ there exist functions $F, \phi \in C^{0,2,2}\left(\Omega\left(\alpha_{0}, \beta_{0}\right), R^{n}\right)$ such that $f_{y} \geq 0$ and $H(f+\phi) \geq 0$ and $H(\phi) \geq 0$.

Then there exist two sequences $\left\{\alpha_{n}(t)\right\}$ and $\left\{\beta_{n}(t)\right\}$ converging uniformly to the solution $x(t)$ of PBVP (2.1) and the convergence is quadratic, that is, there exist positive matrices $K_{3}, K_{4}$ such that for the solution $x(t)$ of PBVP (2.1) in $S\left(\alpha_{0}, \beta_{0}\right)$, the inequalities

$$
\begin{aligned}
& \left|x-\alpha_{n+1}\right|_{0} \leq K_{3}\left|x-\alpha_{n}\right|_{0}^{2}, \\
& \left|\beta_{n+1}-x\right|_{0} \leq K_{4}\left|\beta_{n}-x\right|_{0}^{2}
\end{aligned}
$$

hold, where

$$
|u|_{0}=\max _{s \in[-h, T]}|u(s)|=\left(\max _{s \in[-h, T]}\left|u_{1}(s)\right|, \max _{s \in[-h, T]}\left|u_{2}(s)\right|, \ldots, \max _{s \in[-h, T]}\left|u_{n}(s)\right|\right)^{T} .
$$


Proof In view of $\left(\mathrm{A}_{4.1}\right)$, we see that

$$
\begin{aligned}
f\left(t, x(t), \max _{s \in[t-h, t]} x(s)\right) \geq & f\left(t, y(t), \max _{s \in[t-h, t]} y(s)\right)+f_{x}\left(t, y(t), \max _{s \in[t-h, t]} y(s)\right)(x(t)-y(t)) \\
& +f_{y}\left(t, y(t), \max _{s \in[t-h, t]} y(s)\right)\left(\max _{s \in[t-h, t]} x(s)-\max _{s \in[t-h, t]} y(s)\right) \\
& -\frac{1}{2} \bar{N}\left(|x(t)-y(t)|^{2}+\left|\max _{s \in[t-h, t]} x(s)-\max _{s \in[t-h, t]} y(s)\right|^{2}\right)
\end{aligned}
$$

and

$$
\begin{aligned}
f\left(t, x(t), \max _{s \in[t-h, t]} x(s)\right) \leq & f\left(t, y(t), \max _{s \in[t-h, t]} y(s)\right)+f_{x}\left(t, y(t), \max _{s \in[t-h, t]} y(s)\right)(x(t)-y(t)) \\
& +f_{y}\left(t, y(t), \max _{s \in[t-h, t]} y(s)\right)\left(\max _{s \in[t-h, t]} x(s)-\max _{s \in[t-h, t]} y(s)\right) \\
& +\frac{1}{2} \bar{M}\left(|x(t)-y(t)|^{2}+\left|\max _{s \in[t-h, t]} x(s)-\max _{s \in[t-h, t]} y(s)\right|^{2}\right),
\end{aligned}
$$

where

$$
\begin{aligned}
H(\phi) \leq & \left(\sum_{j=1}^{n}\left|\phi_{x_{j} x}\right|+\sum_{j=1}^{n}\left|\phi_{y_{j} x}\right|\right)|x(t)-y(t)|^{2} \\
& +\left(\sum_{j=1}^{n}\left|\phi_{x_{j} y}\right|+\sum_{j=1}^{n}\left|\phi_{y_{j} y}\right|\right)\left|\max _{s \in[t-h, t]} x(s)-\max _{s \in[t-h, t]} y(s)\right|^{2} \\
\leq & \left(N_{1}+N_{3}\right)|x(t)-y(t)|^{2}+\left(N_{2}+N_{4}\right)\left|\max _{s \in[t-h, t]} x(s)-\max _{s \in[t-h, t]} y(s)\right|^{2} \\
\leq & \bar{N}\left(|x(t)-y(t)|^{2}+\left|\max _{s \in[t-h, t]} x(s)-\max _{s \in[t-h, t]} y(s)\right|^{2}\right)
\end{aligned}
$$

with $\alpha_{0}(t) \leq x(t), y(t) \leq \beta_{0}(t), \sum_{j=1}^{n}\left|\phi_{x_{j} x}\right| \leq N_{1}, \quad \sum_{j=1}^{n}\left|\phi_{x_{j} y}\right| \leq N_{2}, \quad \sum_{j=1}^{n}\left|\phi_{y_{j} x}\right| \leq N_{3}$, $\sum_{j=1}^{n}\left|\phi_{y_{j}}\right| \leq N_{4}$ for $t \in[0, T], N_{1}, N_{2}, N_{3}$, and $N_{4}$ are positive matrices, $\bar{N}=N_{1}+N_{2}+$ $N_{3}+N_{4}$. We have

$$
\begin{aligned}
H(F) \leq & \left(\sum_{j=1}^{n}\left|F_{x_{j} x}\right|+\sum_{j=1}^{n}\left|F_{y_{j} x}\right|\right)|x(t)-y(t)|^{2} \\
& +\left(\sum_{j=1}^{n}\left|F_{x_{j} y}\right|+\sum_{j=1}^{n}\left|F_{y_{j} y}\right|\right)\left|\max _{s \in[t-h, t]} x(s)-\max _{s \in[t-h, t]} y(s)\right|^{2} \\
\leq & \left(M_{1}+M_{3}\right)|x(t)-y(t)|^{2}+\left(M_{2}+M_{4}\right)\left|\max _{s \in[t-h, t]} x(s)-\max _{s \in[t-h, t]} y(s)\right|^{2} \\
\leq & \bar{M}\left(|x(t)-y(t)|^{2}+\left|\max _{s \in[t-h, t]} x(s)-\max _{s \in[t-h, t]} y(s)\right|^{2}\right)
\end{aligned}
$$

with $\alpha_{0}(t) \leq x(t), y(t) \leq \beta_{0}(t), \sum_{j=1}^{n}\left|F_{x_{j} x}\right| \leq M_{1}, \quad \sum_{j=1}^{n}\left|F_{x_{j} y}\right| \leq M_{2}, \quad \sum_{j=1}^{n}\left|F_{y_{j} x}\right| \leq M_{3}$, $\sum_{j=1}^{n}\left|F_{y_{j}}\right| \leq M_{4}$ for $t \in[0, T], M_{1}, M_{2}, M_{3}$, and $M_{4}$ are positive matrices, and $\bar{M}=$ $M_{1}+M_{2}+M_{3}+M_{4}$. 
We consider the singular differential system with 'maxima'

$$
\left\{\begin{aligned}
A x^{\prime}(t)= & f\left(t, \alpha_{0}(t), \max _{s \in[t-h, t]} \alpha_{0}(s)\right)+f_{x}\left(t, \alpha_{0}(t), \max _{s \in[t-h, t]} \alpha_{0}(s)\right)\left(x(t)-\alpha_{0}(t)\right) \\
& +f_{y}\left(t, \alpha_{0}(t), \max _{s \in[t-h, t]} \alpha_{0}(s)\right)\left(\max _{s \in[t-h, t]} x(s)-\max _{s \in[t-h, t]} \alpha_{0}(s)\right) \\
& -\frac{1}{2} \bar{N}\left(\left|x(t)-\alpha_{0}(t)\right|^{2}+\left|\max _{s \in[t-h, t]} x(s)-\max _{s \in[t-h, t]} \alpha_{0}(s)\right|^{2}\right) \\
\equiv & F_{0}\left(t, x(t), \max _{s \in[t-h, t]} x(s)\right), \quad t \in[0, T], \\
x(0)= & x(T), \quad x(t)=x(0), \quad t \in[-h, 0] .
\end{aligned}\right.
$$

Similar to the proof of Theorem 3.1, we can show that $\alpha_{0}(t)$ and $\beta_{0}(t)$ are lower and upper solutions of PBVP (4.3), respectively. Consequently, by Lemma 2.4, there exists a solution $\alpha_{1}(t)$ of PBVP (4.3) such that $\alpha_{0}(t) \leq \alpha_{1}(t) \leq \beta_{0}(t)$ on $[-h, T]$.

Next, we consider the singular differential system with 'maxima'

$$
\left\{\begin{aligned}
A x^{\prime}(t)= & f\left(t, \beta_{0}(t), \max _{s \in[t-h, t]} \beta_{0}(s)\right)+f_{x}\left(t, \beta_{0}(t), \max _{s \in[t-h, t]} \beta_{0}(s)\right)\left(x(t)-\beta_{0}(t)\right) \\
& +f_{y}\left(t, \beta_{0}(t), \max _{s \in[t-h, t]} \beta_{0}(s)\right)\left(\max _{s \in[t-h, t]} x(s)-\max _{s \in[t-h, t]} \beta_{0}(s)\right) \\
& +\frac{1}{2} \bar{M}\left(\left|x(t)-\beta_{0}(t)\right|^{2}+\left|\max _{s \in[t-h, t]} x(s)-\max _{s \in[t-h, t]} \beta_{0}(s)\right|^{2}\right) \\
\equiv & G_{0}\left(t, x(t), \max _{s \in[t-h, t]} x(s)\right), \quad t \in[0, T], \\
x(0)= & x(T), \quad x(t)=x(0), \quad t \in[-h, 0] .
\end{aligned}\right.
$$

Similar to the proof of PBVP (3.4), we see that there exists a solution $\beta_{1}(t)$ of (4.4) such that $\alpha_{1}(t) \leq \beta_{1}(t) \leq \beta_{0}(t)$ on $[-h, T]$.

Now, we shall show that $\alpha_{1}(t)$ and $\beta_{1}(t)$ are lower and upper solutions of PBVP (2.1), respectively. In fact, utilizing the conclusion that $\alpha_{1}(t)$ is a solution of (4.3) and the inequality (4.1), we get

$$
\begin{aligned}
A \alpha_{1}^{\prime}(t)= & f\left(t, \alpha_{0}(t), \max _{s \in[t-h, t]} \alpha_{0}(s)\right) \\
& +f_{x}\left(t, \alpha_{0}(t), \max _{s \in[t-h, t]} \alpha_{0}(s)\right)\left(\alpha_{1}(t)-\alpha_{0}(t)\right) \\
& +f_{y}\left(t, \alpha_{0}(t), \max _{s \in[t-h, t]} \alpha_{0}(s)\right)\left(\max _{s \in[t-h, t]} \alpha_{1}(s)-\max _{s \in[t-h, t]} \alpha_{0}(s)\right) \\
& -\frac{1}{2} \bar{N}\left(\left|\alpha_{1}(t)-\alpha_{0}(t)\right|^{2}+\left|\max _{s \in[t-h, t]} \alpha_{1}(s)-\max _{s \in[t-h, t]} \alpha_{0}(s)\right|^{2}\right) \\
\leq & f\left(t, \alpha_{1}(t), \max _{s \in[t-h, t]} \alpha_{1}(s)\right), \quad t \in[0, T], \\
\alpha_{1}(0)= & \alpha_{1}(T), \quad \alpha_{1}(t)=\alpha_{1}(0), \quad t \in[-h, 0] .
\end{aligned}
$$

It proves that $\alpha_{1}(t)$ is a lower solution of PBVP $(2.1)$ on $[-h, T]$. Analogously, we see that $\beta_{1}(t)$ is an upper solution of PBVP (2.1). Consequently, these results yield

$$
\alpha_{0}(t) \leq \alpha_{1}(t) \leq \beta_{1}(t) \leq \beta_{0}(t), \quad t \in[-h, T]
$$

Proceeding as before, we can get

$$
\alpha_{n}(t) \leq \alpha_{n+1}(t) \leq \beta_{n+1}(t) \leq \beta_{n}(t), \quad t \in[-h, T],
$$


where $\alpha_{n+1}(t)$ and $\beta_{n+1}(t)$ are solutions of the singular differential systems with 'maxima',

$$
\left\{\begin{aligned}
A x^{\prime}(t)= & f\left(t, \alpha_{n}(t), \max _{s \in[t-h, t]} \alpha_{n}(s)\right)+f_{x}\left(t, \alpha_{n}(t), \max _{s \in[t-h, t]} \alpha_{n}(s)\right)\left(x(t)-\alpha_{n}(t)\right) \\
& +f_{y}\left(t, \alpha_{n}(t), \max _{s \in[t-h, t]} \alpha_{n}(s)\right)\left(\max _{s \in[t-h, t]} x(s)-\max _{s \in[t-h, t]} \alpha_{n}(s)\right) \\
& -\frac{1}{2} \bar{N}\left(\left|x(t)-\alpha_{n}(t)\right|^{2}+\left|\max _{s \in[t-h, t]} x(s)-\max _{s \in[t-h, t]} \alpha_{n}(s)\right|^{2}\right) \\
\equiv & F_{n}\left(t, x(t), \max _{s \in[t-h, t]} x(s)\right), \quad t \in[0, T], \\
x(0)= & x(T), \quad x(t)=x(0), \quad t \in[-h, 0]
\end{aligned}\right.
$$

and

$$
\left\{\begin{aligned}
A x^{\prime}(t)= & f\left(t, \beta_{n}(t), \max _{s \in[t-h, t]} \beta_{n}(s)\right)+f_{x}\left(t, \beta_{n}(t), \max _{s \in[t-h, t]} \beta_{n}(s)\right)\left(x(t)-\beta_{n}(t)\right) \\
& +f_{y}\left(t, \beta_{n}(t), \max _{s \in[t-h, t]} \beta_{n}(s)\right)\left(\max _{s \in[t-h, t]} x(s)-\max _{s \in[t-h, t]} \beta_{n}(s)\right) \\
& +\frac{1}{2} \bar{M}\left(\left|x(t)-\beta_{n}(t)\right|^{2}+\left|\max _{s \in[t-h, t]} x(s)-\max _{s \in[t-h, t]} \beta_{n}(s)\right|^{2}\right) \\
\equiv & G_{n}\left(t, x(t), \max _{s \in[t-h, t]} x(s)\right), \quad t \in[0, T], \\
x(0)= & x(T), \quad x(t)=x(0), \quad t \in[-h, 0] .
\end{aligned}\right.
$$

Using mathematical induction, we can show that, for all $n$,

$$
\alpha_{0}(t) \leq \alpha_{1}(t) \leq \cdots \leq \alpha_{n}(t) \leq \beta_{n}(t) \leq \cdots \leq \beta_{1}(t) \leq \beta_{0}(t), \quad t \in[-h, T] .
$$

It is clear that both sequences $\left\{\alpha_{n}(t)\right\}$ and $\left\{\beta_{n}(t)\right\}$ are uniformly bounded and equicontinuous on $[-h, T]$. In view of the Ascoli-Arzela theorem, we have

$$
\begin{aligned}
& \lim _{n \rightarrow \infty} \alpha_{n}(t)=\rho(t), \\
& \lim _{n \rightarrow \infty} \beta_{n}(t)=r(t) .
\end{aligned}
$$

It remains to show the quadratic convergence. For this purpose, set

$$
a_{n+1}(t)=x(t)-\alpha_{n+1}(t) \geq 0, \quad t \in[-h, T] .
$$

Case 1. $t \in[-h, 0], a_{n+1}(t)=a_{n+1}(0)=a_{n+1}(T)$.

Case 2. $t \in[0, T]$. Using the assumption $f_{y} \geq 0$ and the inequality (4.2), we obtain

$$
\begin{aligned}
A a_{n+1}{ }^{\prime}(t) \leq & f\left(t, \alpha_{n}(t), \max _{s \in[t-h, t]} \alpha_{n}(s)\right)+f_{x}\left(t, \alpha_{n}(t), \max _{s \in[t-h, t]} \alpha_{n}(s)\right)\left(x(t)-\alpha_{n}(t)\right) \\
& +f_{y}\left(t, \alpha_{n}(t) \max _{s \in[t-h, t]} \alpha_{n}(s)\right)\left(\max _{s \in[t-h, t]} x(s)-\max _{s \in[t-h, t]} \alpha_{n}(s)\right) \\
& +\frac{1}{2} \bar{M}\left(\left|x(t)-\alpha_{n}(t)\right|^{2}+\left|\max _{s \in[t-h, t]} x(s)-\max _{s \in[t-h, t]} \alpha_{n}(s)\right|^{2}\right) \\
& -\left[f\left(t, \alpha_{n}(t) \max _{s \in[t-h, t]} \alpha_{n}(s)\right)+f_{x}\left(t, \alpha_{n}(t) \max _{s \in[t-h, t]} \alpha_{n}(s)\right)\left(\alpha_{n+1}(t)-\alpha_{n}(t)\right)\right. \\
& +f_{y}\left(t, \alpha_{n}(t) \max _{s \in[t-h, t]} \alpha_{n}(s)\right)\left(\max _{s \in[t-h, t]} \alpha_{n+1}(s)-\max _{s \in[t-h, t]} \alpha_{n}(s)\right) \\
& \left.-\frac{1}{2} \bar{N}\left(\left|\alpha_{n+1}(t)-\alpha_{n}(t)\right|^{2}+\left|\max _{s \in[t-h, t]} \alpha_{n+1}(s)-\max _{s \in[t-h, t]} \alpha_{n}(s)\right|^{2}\right)\right] \\
\leq & f_{x}\left(t, \alpha_{n}(t) \max _{s \in[t-h, t]} \alpha_{n}(s)\right)\left(x(t)-\alpha_{n+1}(t)\right)
\end{aligned}
$$




$$
\begin{aligned}
& +f_{y}\left(t, \alpha_{n}(t), \max _{s \in[t-h, t]} \alpha_{n}(s)\right) \max _{s \in[t-h, t]}\left(x(s)-\alpha_{n+1}(s)\right) \\
& +\frac{1}{2}(\bar{M}+\bar{N})\left(\left|x(t)-\alpha_{n}(t)\right|^{2}+\left|\max _{s \in[t-h, t]} x(s)-\max _{s \in[t-h, t]} \alpha_{n}(s)\right|^{2}\right) \\
\leq & -M(\bar{t}) a_{n+1}(t)-N(\bar{t}) \max _{s \in[t-h, t]} a_{n+1}(s)+(\bar{M}+\bar{N}) \max _{s \in[t-h, t]}\left|a_{n}(s)\right|^{2} \\
\leq & -M(\bar{t}) a_{n+1}(t)-N(\bar{t}) \max _{s \in[0, T]} a_{n+1}(s)+(\bar{M}+\bar{N}) \max _{s \in[0, T]}\left|a_{n}(s)\right|^{2} .
\end{aligned}
$$

Then we obtain

$$
\begin{aligned}
& A a_{n+1}{ }^{\prime}(t) \leq-M(\bar{t}) a_{n+1}(t)-N(\bar{t}) \max _{s \in[0, T]} a_{n+1}(s)+(\bar{M}+\bar{N}) \max _{s \in[0, T]}\left|a_{n}(s)\right|^{2}, \quad t \in[0, T], \\
& a_{n+1}(0)=a_{n+1}(T), \quad a_{n+1}(t)=a_{n+1}(0), \quad t \in[-h, 0] .
\end{aligned}
$$

According to Lemma 2.1, we obtain $a_{n+1}(t) \leq u(t)$ on $[0, T]$, where $u(t)$ is the solution of

$$
\begin{aligned}
& A u^{\prime}+M(\bar{t}) u=-N(\bar{t}) \max _{s \in[0, T]} a_{n+1}(s)+(\bar{M}+\bar{N}) \max _{s \in[0, T]}\left|a_{n}(s)\right|^{2}, \quad t \in[0, T], \\
& u(0)=u(T), \quad u(t)=u(0), \quad t \in[-h, 0] .
\end{aligned}
$$

Thus, using the expression of $x(t)$ in Lemma 2.2 and taking suitable estimates, we arrive at

$$
\left|a_{n+1}(t)\right|_{0} \leq K_{3}\left|a_{n}(t)\right|_{0}^{2}
$$

where $K_{3}$ is a positive matrix.

Similarly, consider

$$
b_{n+1}(t)=\beta_{n+1}(t)-x(t) \geq 0, \quad t \in[-h, T] .
$$

Case 1. $t \in[-h, 0], b_{n+1}(t)=b_{n+1}(0)=b_{n+1}(T)$.

Case 2. $t \in[0, T]$. It follows from the assumption $f_{y} \geq 0$ and the inequality (4.1) that

$$
\begin{aligned}
A b_{n+1}{ }^{\prime}(t) \leq & f\left(t, \beta_{n}(t), \max _{s \in[t-h, t]} \beta_{n}(s)\right)+f_{x}\left(t, \beta_{n}(t), \max _{s \in[t-h, t]} \beta_{n}(s)\right)\left(\beta_{n+1}(t)-\beta_{n}(t)\right) \\
& +f_{y}\left(t, \beta_{n}(t), \max _{s \in[t-h, t]} \beta_{n}(s)\right)\left(\max _{s \in[t-h, t]} \beta_{n+1}(s)-\max _{s \in[t-h, t]} \beta_{n}(s)\right) \\
& +\frac{1}{2} \bar{M}\left(\left|\beta_{n+1}(t)-\beta_{n}(t)\right|^{2}+\left|\max _{s \in[t-h, t]} \beta_{n+1}(s)-\max _{s \in[t-h, t]} \beta_{n}(s)\right|^{2}\right) \\
& -\left[f\left(t, \beta_{n}(t) \max _{s \in[t-h, t]} \beta_{n}(s)\right)+f_{x}\left(t, \beta_{n}(t), \max _{s \in[t-h, t]} \beta_{n}(s)\right)\left(x(t)-\beta_{n}(t)\right)\right. \\
& +f_{y}\left(t, \beta_{n}(t), \max _{s \in[t-h, t]} \beta_{n}(s)\right)\left(\max _{s \in[t-h, t]} x(s)-\max _{s \in[t-h, t]} \beta_{n}(s)\right) \\
& \left.-\frac{1}{2} \bar{N}\left(\left|x(t)-\beta_{n}(t)\right|^{2}+\left|\max _{s \in[t-h, t]} x(s)-\max _{s \in[t-h, t]} \beta_{n}(s)\right|^{2}\right)\right] \\
\leq & f_{x}\left(t, \beta_{n}(t), \max _{s \in[t-h, t]} \beta_{n}(s)\right)\left(\beta_{n+1}(t)-x(t)\right)
\end{aligned}
$$




$$
\begin{aligned}
& +f_{y}\left(t, \beta_{n}(t), \max _{s \in[t-h, t]} \beta_{n}(s)\right) \max _{s \in[t-h, t]}\left(\beta_{n+1}(s)-x(s)\right) \\
& +\frac{1}{2}(\bar{M}+\bar{N})\left(\left|\beta_{n}(t)-x(t)\right|^{2}+\left|\max _{s \in[t-h, t]} \beta_{n}(s)-\max _{s \in[t-h, t]} x(s)\right|^{2}\right) \\
\leq & -M(\bar{t}) b_{n+1}(t)-N(\bar{t}) \max _{s \in[t-h, t]} b_{n+1}(s)+(\bar{M}+\bar{N}) \max _{s \in[t-h, t]}\left|b_{n}(s)\right|^{2} \\
\leq & -M(\bar{t}) b_{n+1}(t)-N(\bar{t}) \max _{s \in[0, T]} b_{n+1}(s)+(\bar{M}+\bar{N}) \max _{s \in[0, T]}\left|b_{n}(s)\right|^{2} .
\end{aligned}
$$

Then we obtain

$$
\begin{aligned}
& A b_{n+1}{ }^{\prime}(t) \leq-M(\bar{t}) b_{n+1}(t)-N(\bar{t}) \max _{s \in[0, T]} b_{n+1}(s)+(\bar{M}+\bar{N}) \max _{s \in[0, T]}\left|b_{n}(s)\right|^{2}, \quad t \in[0, T], \\
& b_{n+1}(0)=b_{n+1}(T), \quad b_{n+1}(t)=b_{n+1}(0), \quad t \in[-h, 0] .
\end{aligned}
$$

According to Lemma 2.1, we obtain $b_{n+1}(t) \leq u(t)$ on $[0, T]$, where $u(t)$ is the solution of

$$
\begin{aligned}
& A u^{\prime}+M(\bar{t}) u=-N(\bar{t}) \max _{s \in[0, T]} b_{n+1}(s)+(\bar{M}+\bar{N}) \max _{s \in[0, T]}\left|b_{n}(s)\right|^{2}, \quad t \in[0, T], \\
& u(0)=u(T), \quad u(t)=u(0), \quad t \in[-h, 0] .
\end{aligned}
$$

Thus, utilizing the expression of $x(t)$ in Lemma 2.2, we obtain after taking suitable estimates

$$
\left|b_{n+1}\right|_{0} \leq K_{4}\left|b_{n}(t)\right|_{0}^{2}
$$

Hence, the convergence is quadratic. The proof of the theorem is complete.

\section{Competing interests}

The authors declare that they have no competing interests.

\section{Authors' contributions}

All authors completed the paper together. All authors read and approved the final manuscript.

\section{Author details}

${ }^{1}$ College of Electronic and Information Engineering, Hebei University, Baoding, 071002, China. ${ }^{2}$ College of Mathematics and Information Science, Hebei University, Baoding, 071002, China.

\section{Acknowledgements}

This paper is supported by the National Natural Science Foundation of China (11271106) and the Natural Science Foundation of Hebei Province of China (A2013201232).

Received: 14 August 2015 Accepted: 23 October 2015 Published online: 31 October 2015

\section{References}

1. Bellman, R, Kalaba, R: Quasilinearization and Nonlinear Boundary Value Problems. Elsevier, New York (1965)

2. Lakshmikantham, V, Vatsala, AS: Generalized Quasilinearization for Nonlinear Problems. Kluwer Academic, Dordrecht (1998)

3. Lakshmikantham, V, Koksal, S: Monotone Flows and Rapid Convergence for Nonlinear Partial Differential Equations. Taylor \& Francis, London (2003)

4. Laksmikantham, V, Leela, S, Drici, Z, McRae, FA: Theory of Causal Differential Equations. Atlantis Press, Paris (2009)

5. Ahmad, B, Khan, RA, Sivasundaram, S: Generalized quasilinearization method for nonlinear functional differential equations. J. Appl. Math. Stoch. Anal. 16, 33-43 (2003)

6. Drici, Z, McRae, FA, Devi, JV: Quasilinearization for functional differential equations with retardation and anticipation. Nonlinear Anal. 70, 1763-1775 (2009)

7. Bainov, DD, Hristova, SG: The method of quasilinearization for the periodic boundary value problem for systems of impulsive differential equations. Appl. Math. Comput. 117, $73-85$ (2001) 
8. Ahmad, B, Alsaedi, A: An extended method of quasilinearization for nonlinear impulsive differential equations with a nonlinear three-point boundary condition. Electron. J. Qual. Theory Differ. Equ. 2007, 1 (2007)

9. Devi, JV: Generalized monotone method for periodic boundary value problems of Caputo fractional differential equations. Commun. Appl. Anal. 12(4), 399-406 (2008)

10. Devi, JV, McRae, FA, Drici, Z: Generalized quasilinearization for fractional differential equations. Comput. Math. Appl. 59, 1057-1062 (2010)

11. Wang, $P G, H o u, Y$ : Generalized quasilinearization for the system of fractional differential equations. J. Funct. Spaces Appl. 2013, Article ID 793263 (2013)

12. Rosenbrock, HH: Structural properties of linear dynamical systems. Int. J. Control 200, 191-202 (1974)

13. Abd-Ellateef Kamar, AR, Attia, GM, Vajravelu, K, Mosaad, M: Generalized quasilinearization for singular system of differential equations. Appl. Math. Comput. 114, 69-74 (2000)

14. O'Regan, D, El-Gebeily, M: Existence, upper and lower solutions and quasilinearization for singular differential equations. IMA J. Appl. Math. 73, 323-344 (2008)

15. El-Gebeily, M, O'Regan, D: A quasilinearization method for a class of second order singular nonlinear differential equations with nonlinear boundary conditions. Nonlinear Anal., Real World Appl. 8(1), 174-186 (2007)

16. El-Gebeily, M, O'Regan, D: Upper and lower solutions and quasilinearization for a class of second order singular nonlinear differential equations with nonlinear boundary conditions. Nonlinear Anal., Real World Appl. 8(2), 636-645 (2007)

17. Bainov, D, Hristova, S: Differential Equations with Maxima. CRC Press, Boca Raton (2011)

18. Agarwal, RP, Hristova, S: Quasilinearization for initial value problems involving differential equations with 'maxima'. Math. Comput. Model. 55, 2096-2105 (2012)

\section{Submit your manuscript to a SpringerOpen ${ }^{\circ}$ journal and benefit from:}

- Convenient online submission

Rigorous peer review

- Immediate publication on acceptance

Open access: articles freely available online

- High visibility within the field

- Retaining the copyright to your article 